\title{
Force Generated by a Magnetic Field Applied on a Circular Conductive Turn Rotated in Two Cartesian Axes
}

\author{
Romualdo S. Silva \\ Department of Physics, Federal University of Sergipe, São Cristóvão, Brazil
}

Email address:

romu.fisica@gmail.com

To cite this article:

Romualdo S. Silva. Force Generated by a Magnetic Field Applied on a Circular Conductive Turn Rotated in Two Cartesian Axes. American Journal of Electromagnetics and Applications. Vol. 5, No. 2, 2017, pp. 20-23. doi: 10.11648/j.ajea.20170502.12

Received: December 7, 2016; Accepted: January 16, 2017; Published: December 19, 2017

\begin{abstract}
This article presents a very detailed resolution of a non-trivial problem in Electromagnetic Theory. The problem basically consists of a circular conducting loop of radius $\mathrm{R}$, which has a current $\mathrm{I}$, and is located with its center at the origin of the Cartesian coordinate system. It is rotated with respect to the normal to its plane with angles of $\theta_{0}$ and $\varphi_{0}$ in spherical coordinates, in addition, there is an applied External Magnetic Field. The forces generated by the magnetic field in all directions were calculated without approximations, where in the $\mathrm{z}$ direction the force is zero, as expected.
\end{abstract}

Keywords: Magnetic Field, Circular Loop, Rotation, Force

\section{Introduction}

Classical electromagnetic theory, together with classical mechanics and quantum mechanics, constitute a core of extremely important disciplines for undergraduate and graduate students in physics [1-3]. The mathematical tool used in these courses usually involves vector calculations, ordinary and partial differential equations, Fourier series, Laplace transformations, among others [4-6].

Mechanics tells us as a system that is subject to a certain Force. We know that there are for the moment only the fundamental forces in Physics, which are: Strong, Electromagnetic, Weak and Gravitational, are written from stronger to weak, respectively [7, 8].

Strong forces are the ones that keep protons and neutrons attached to the atoms, they have an extremely short range, but they are a hundred times stronger than the electric forces. The Weak force, which is of the radioactive decay, not only has a short range, but is also much weaker than the electromagnetic. As for Gravitational, it is such a despicable one, that it is only due to great concentrations of masses, like the Earth and the Sun, that we can even perceive it.

The laws of electrodynamics were gradually discovered by Franklin, Coulomb, Ampère, Faraday, among others. But who actually completed this task, compacting the equations in a way, was the famous Maxwell [9, 10].
In this way, when we talk about solving problems, many students present difficulties, due to the degree of mathematical complexity present in the exercises, ending up often failing to solve some problems.

The main objective of this work is to solve, in a systematic and unprecedented way, an intriguing and quite interesting problem of electromagnetic theory, which is not so trivial but with a certain mathematical capacity we can solve the problem.

\section{Calculation of Forces Acting on the Loop}

The problem basically consists of a circular conducting loop of radius $\mathrm{R}$, which has a current $\mathrm{I}$, and is located with its center at the origin of the Cartesian coordinate system. It is rotated with respect to the normal to its plane with angles of $\theta_{0}$ and $\varphi_{0}$ in spherical coordinates, as can be observed in Figure 1. In addition, there is an applied external magnetic field, written as follows $\vec{B}(\overrightarrow{\mathrm{x}})=B_{0}(1+\eta y) \hat{i}+B_{0}(1+\eta x) \hat{j}$

We intend to calculate then the resulting force acting on the loop without making any approximation. We know that by the Law of Biot-Savart [7, 11], when a current with a density $\vec{J}(\overrightarrow{\mathrm{x}})$ is in an external magnetic field with a magnetic flux density $\vec{B}(\overrightarrow{\mathrm{x}})$, The law of fundamental force says that the 
total force on the current distribution is given by [1].

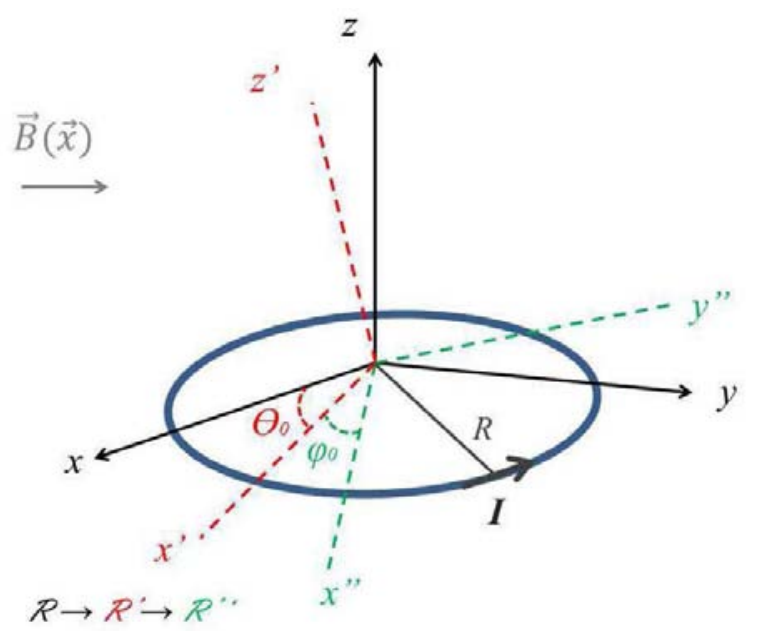

Figure 1. Sketch of the problem of a circular conductive loop, rotated on two Cartesian axes, with an applied external field.

$$
\vec{F}=\frac{1}{c} \int \vec{J}(\overrightarrow{\mathrm{x}}) \otimes \vec{B}(\overrightarrow{\mathrm{x}}) d^{3} \overrightarrow{\mathrm{x}}
$$

Since this problem is not so simple, since we have a rotation of coordinates in two Cartesian axes, we must make a rotation of coordinates as follows: $R(x, y, z) \rightarrow R^{\prime}\left(x^{\prime}, y^{\prime}, z^{\prime}\right) \rightarrow$ $R "(x,, y ", z ")$.

In this way the new coordinates can be written as:

$$
\left\{\begin{array}{l}
x^{\prime}=\cos \varphi_{0} x+\operatorname{sen} \varphi_{0} y \\
y^{\prime}=-\operatorname{sen} \varphi_{0} x+\cos \varphi_{0} y \\
z^{\prime}=z
\end{array}\right.
$$

$$
\left\{\begin{array}{l}
x^{\prime \prime}=\cos \theta_{0} x^{\prime}-\operatorname{sen} \theta_{0} z^{\prime} \\
y^{\prime \prime}=y^{\prime} \\
z^{\prime \prime}=\operatorname{sen} \theta_{0} x^{\prime}+\cos \theta_{0} z^{\prime}
\end{array}\right.
$$

To facilitate the understanding of the calculation, let's write the coordinates in matrix form [12], as follows:

$$
\begin{aligned}
& \left(\begin{array}{l}
x^{\prime} \\
y^{\prime} \\
z^{\prime}
\end{array}\right)=\left(\begin{array}{ccc}
\cos \varphi_{0} & \operatorname{sen} \varphi_{0} & 0 \\
-\operatorname{sen} \varphi_{0} & \cos \varphi_{0} & 0 \\
0 & 0 & 1
\end{array}\right)\left(\begin{array}{l}
x \\
y \\
z
\end{array}\right) \\
& \left(\begin{array}{l}
x^{\prime \prime} \\
y^{\prime \prime} \\
z^{\prime \prime}
\end{array}\right)=\left(\begin{array}{ccc}
\cos \theta_{0} & 0 & -\operatorname{sen} \theta_{0} \\
0 & 1 & 0 \\
\operatorname{sen} \theta_{0} & 0 & \cos \theta_{0}
\end{array}\right)\left(\begin{array}{l}
x^{\prime} \\
y^{\prime} \\
z^{\prime}
\end{array}\right)
\end{aligned}
$$

To find the coordinates $(x, y, z)$ we have to multiply the matrix (4) by the matrix (3), and write the transposed matrix, that is:

$$
\begin{aligned}
& \left(\begin{array}{c}
x^{\prime \prime} \\
y^{\prime \prime} \\
z^{\prime \prime}
\end{array}\right)\left(\begin{array}{l}
x^{\prime} \\
y^{\prime} \\
z^{\prime}
\end{array}\right)=\left(\begin{array}{ccc}
\cos \theta_{0} & 0 & -\operatorname{sen} \theta_{0} \\
0 & 1 & 0 \\
\operatorname{sen} \theta_{0} & 0 & \cos \theta_{0}
\end{array}\right) \times \\
& \times\left(\begin{array}{ccc}
\cos \varphi_{0} & \operatorname{sen} \varphi_{0} & 0 \\
-\operatorname{sen} \varphi_{0} & \cos \varphi_{0} & 0 \\
0 & 0 & 1
\end{array}\right)\left(\begin{array}{l}
x \\
y \\
z
\end{array}\right) \\
& \left(\begin{array}{c}
x^{\prime \prime} \\
y^{\prime \prime} \\
z^{\prime \prime}
\end{array}\right)\left(\begin{array}{c}
x^{\prime} \\
y^{\prime} \\
z^{\prime}
\end{array}\right)= \\
& \left(\begin{array}{ccc}
\cos \theta_{0} \cos \varphi_{0} & \cos \theta_{0} \operatorname{sen} \varphi_{0} & -\operatorname{sen} \theta_{0} \\
-\operatorname{sen} \varphi_{0} & \cos \varphi_{0} & 0 \\
\operatorname{sen} \theta_{0} \cos \varphi_{0} & \operatorname{sen} \theta_{0} \operatorname{sen} \varphi_{0} & \cos \theta_{0}
\end{array}\right)\left(\begin{array}{l}
x \\
y \\
z
\end{array}\right)
\end{aligned}
$$

Consequently, through this matrix we can write the unitary verses, being:

$$
\left\{\begin{array}{l}
\hat{i}^{\prime \prime}=\cos \theta_{0} \cos \varphi_{0} \hat{i}+\cos \theta_{0} \operatorname{sen} \varphi_{0} \hat{j}-\operatorname{sen} \theta_{0} \hat{k} \\
\hat{j}^{\prime \prime}=\operatorname{sen} \varphi_{0} \hat{i}+\cos \varphi_{0} \hat{j} \\
\hat{\mathrm{k}}^{\prime \prime}=\operatorname{sen} \theta_{0} \cos \varphi_{0} \hat{i}+\operatorname{sen} \theta_{0} \operatorname{sen} \varphi_{0} \hat{j}+\cos \theta_{0} \hat{k}
\end{array}\right.
$$

Writing the transposed matrix, which basically means replacing what was column in line and what was line turns column, we get then:

$$
\begin{gathered}
\left(\begin{array}{l}
x \\
y \\
z
\end{array}\right)= \\
\left(\begin{array}{ccc}
\cos \theta_{0} \cos \varphi_{0} & -\operatorname{sen} \varphi_{0} & \operatorname{sen} \theta_{0} \cos \varphi_{0} \\
\cos \theta_{0} \operatorname{sen} \varphi_{0} & \cos \varphi_{0} & \operatorname{sen} \theta_{0} \operatorname{sen} \varphi_{0} \\
-\operatorname{sen} \theta_{0} & 0 & \cos \theta_{0}
\end{array}\right)\left(\begin{array}{l}
x^{\prime \prime} \\
y^{\prime \prime} \\
z^{\prime \prime}
\end{array}\right)
\end{gathered}
$$

Finally, the original coordinates we are looking for can be written as:

$$
\left\{\begin{array}{l}
x=\cos \theta_{0} \cos \varphi_{0} x^{\prime \prime}-\operatorname{sen} \varphi_{0} y^{\prime \prime}+\operatorname{sen} \theta_{0} \cos \varphi_{0} z^{\prime \prime} \\
y=\cos \theta_{0} \operatorname{sen} \varphi_{0} x^{\prime \prime}+\cos \varphi_{0} y^{\prime \prime}+\operatorname{sen} \theta_{0} \operatorname{sen} \varphi_{0} z^{\prime \prime} \\
z=-\operatorname{sen} \theta_{0} x^{\prime \prime}+\cos \theta_{0} z^{\prime \prime}
\end{array}\right.
$$

Now that we have already determined the coordinates (x, y, $\mathrm{z}$ ), our problem consists of calculating the integral of equation 1 , in order to find the force. First we determine the current distribution $\vec{J}(\vec{x}) d^{3} \vec{x}$, and then determine the vector product between the current density $\vec{J}(\vec{x})$ and the magnetic flux $\vec{B}(\vec{x})$. 


$$
\vec{J}(\overrightarrow{\mathrm{x}}) d^{3} \overrightarrow{\mathrm{x}}=I d \vec{l}=\operatorname{IRd} \varphi \hat{\varphi}
$$

But knowing that $\hat{\varphi}=-\operatorname{sen} \varphi \hat{i} "+\cos \varphi \hat{j} "$, then eq. (10) is:

$$
\vec{J}(\overrightarrow{\mathrm{x}}) d^{3} \overrightarrow{\mathrm{x}}=\operatorname{IRd} \varphi\left[-\operatorname{sen} \varphi \hat{i} "+\cos \varphi \hat{j}^{\prime \prime}\right]
$$

From eq. (7), we take that the unitary verses in the $\mathrm{x}$ and $\mathrm{y}$ direction are $\hat{i}^{\prime \prime}=\cos \theta_{0} \cos \varphi_{0} \hat{i}+\cos \theta_{0} \operatorname{sen} \varphi_{0} \hat{j}-\operatorname{sen} \theta_{0} \hat{k}$, and $\hat{j}^{\prime \prime}=\operatorname{sen} \varphi_{0} \hat{i}+\cos \varphi_{0} \hat{j}$, and replacing in (11), is:

$$
\begin{gathered}
\vec{J}(\overrightarrow{\mathrm{x}}) d^{3} \overrightarrow{\mathrm{x}}=\operatorname{IRd} \varphi \times \\
\times\left[-\operatorname{sen} \varphi\left(\cos \theta_{0} \cos \varphi_{0} \hat{i}+\cos \theta_{0} \operatorname{sen} \varphi_{0} \hat{j}-\operatorname{sen} \theta_{0} \hat{k}\right)+\right. \\
\left.+\cos \varphi\left(\operatorname{sen} \varphi_{0} \hat{i}+\cos \varphi_{0} \hat{j}\right)\right] \\
\vec{J}(\overrightarrow{\mathrm{x}}) d^{3} \overrightarrow{\mathrm{x}}=\operatorname{IRd} \varphi \times \\
\times\left[\left(-\operatorname{sen} \varphi \cos \theta_{0} \cos \varphi_{0}-\cos \varphi \operatorname{sen} \varphi_{0}\right) \hat{i}+\right. \\
\left.\left(-\operatorname{sen} \varphi \cos \theta_{0} \operatorname{sen} \varphi_{0}+\cos \varphi \cos \varphi_{0}\right) \hat{j}+\left(\operatorname{sen} \varphi \operatorname{sen} \theta_{0}\right) \hat{k}\right]
\end{gathered}
$$

The magnetic field is written as $\vec{B}(\overrightarrow{\mathrm{x}})=B_{0}(1+\eta y) \hat{i}+B_{0}(1+\eta x) \hat{j}$, but we already know the components $\mathrm{x}$ and $\mathrm{y}$, as shown in eq. (9), in this way we can do the substitution and find that:

$$
\begin{aligned}
\vec{B}(\overrightarrow{\mathrm{x}}) & =B_{0}\left[1+\eta\left(\cos \theta_{0} \operatorname{sen} \varphi_{0} x^{\prime \prime}+\cos \varphi_{0} y^{\prime \prime}\right)\right] \hat{i}+ \\
& +B_{0}\left[1+\eta\left(\cos \theta_{0} \cos \varphi_{0} x^{\prime \prime}-\operatorname{sen} \varphi_{0} y^{\prime \prime}\right)\right] \hat{j}
\end{aligned}
$$

But in spherical coordinates $x^{\prime \prime}=R \cos \varphi$ and $\mathrm{y}^{\prime \prime}=R \operatorname{sen} \varphi$, soon:

$$
\begin{gathered}
\vec{B}(\overrightarrow{\mathrm{x}})= \\
B_{0}\left[1+\eta R\left(\cos \theta_{0} \operatorname{sen} \varphi_{0} \cos \varphi+\cos \varphi_{0} \operatorname{sen} \varphi\right)\right] \hat{i}+ \\
+B_{0}\left[1+\eta R\left(\cos \theta_{0} \cos \varphi_{0} \cos \varphi-\operatorname{sen} \varphi_{0} \operatorname{sen} \varphi\right)\right] \hat{j}
\end{gathered}
$$

Now we replace (13) and (15) in eq. (1), and we find that the force $\vec{F}$ in the loop will then be:

$$
\begin{aligned}
& \vec{F}=\frac{1}{c} \int_{0}^{2 \pi} x \\
& \times\left\{\begin{array}{l}
\operatorname{IR}\left[\left(-\operatorname{sen} \varphi \cos \theta_{0} \cos \varphi_{0}-\cos \varphi \operatorname{sen} \varphi_{0}\right) \hat{i}+\right. \\
+\left(-\operatorname{sen} \varphi \cos \theta_{0} \operatorname{sen} \varphi_{0}+\cos \varphi \cos \varphi_{0}\right) \hat{j}+ \\
\left.+\left(\operatorname{sen} \varphi \operatorname{sen} \theta_{0}\right) \hat{k}\right]
\end{array}\right\} \\
& \otimes\left\{\begin{array}{l}
B_{0}\left[1+\eta R\left(\cos \theta_{0} \operatorname{sen} \varphi_{0} \cos \varphi+\cos \varphi_{0} \operatorname{sen} \varphi\right)\right] \hat{i}+ \\
+B_{0}\left[1+\eta R\left(\cos \theta_{0} \cos \varphi_{0} \cos \varphi-\operatorname{sen} \varphi_{0} \operatorname{sen} \varphi\right)\right] \hat{j}
\end{array}\right\} d \varphi
\end{aligned}
$$

Decomposing the forces we will have:

$$
F_{t}=F_{x}+F_{y}+F_{z}
$$

At where,

$$
F_{x}=\frac{1}{c} \int\left(-J_{z}\right) \otimes\left(B_{y}\right) d^{3} \overrightarrow{\mathrm{x}}
$$

$$
\begin{gathered}
F_{x}=\frac{I R}{c} \int_{0}^{2 \pi}\left[-\left(\operatorname{sen} \varphi \operatorname{sen} \theta_{0}\right)\right] \times \\
\times\left[B _ { 0 } \left(1+\eta R\left\{\cos \theta_{0} \cos \varphi_{0} \cos \varphi+\right.\right.\right. \\
\left.\left.\left.-\cos \varphi_{0} \cos \varphi\right\}\right)\right] d \varphi \\
F_{x}=\frac{I R}{c} \eta R B_{0} \int_{0}^{2 \pi}\left[-\operatorname{sen} \varphi \operatorname{sen} \theta_{0} \cos \theta_{0} \cos \varphi_{0} \cos \varphi+\right. \\
\left.+\operatorname{sen}^{2} \varphi \operatorname{sen} \theta_{0} \operatorname{sen} \varphi_{0}\right] d \varphi
\end{gathered}
$$

Remembering that:

$$
\int_{0}^{2 \pi} \operatorname{sen} \varphi \cos \varphi d \varphi=0 \mathrm{e} \int_{0}^{2 \pi} \operatorname{sen}^{2} \varphi d \varphi=\int_{0}^{2 \pi} \cos ^{2} \varphi d \varphi=\pi
$$

soon:

$$
F_{x}=\frac{I R^{2}}{c} \eta \pi B_{0} \operatorname{sen} \theta_{0} \operatorname{sen} \varphi_{0}
$$

Applying the same reasoning taken in (18), we will have that forces $F_{y}$ e $F_{z}$, will respectively:

$$
\left\{\begin{array}{l}
F_{y}=\frac{1}{c} \int\left(J_{z}\right) \otimes\left(B_{x}\right) d^{3} \overrightarrow{\mathrm{x}} \\
F_{z}=\frac{1}{c} \int\left(J_{x}\right) \otimes\left(B_{y}\right) d^{3} \overrightarrow{\mathrm{x}}
\end{array}\right.
$$

and therefore,

$$
\begin{gathered}
F_{y}=\frac{I R^{2}}{c} \eta \pi B_{0} \operatorname{sen} \theta_{0} \cos \varphi_{0} \\
F_{z}=0
\end{gathered}
$$

As already expected, the force in the z-direction is zero, since the applied external magnetic field has only components in the $\mathrm{x}$ and $\mathrm{y}$ directions, so we will only have force components in these same directions.

\section{Conclusion}

Through this work it was possible to develop and solve a problem of electromagnetic theory consisting of a circular conductive loop with a current I that is rotated in two axes of its Cartesian plane with respect to its normal and still has an external magnetic field. It was found that the force in the z-direction is zero, as expected, since the external field has only components in the $\mathrm{x}$ and $\mathrm{y}$ direction.

It is hoped that this work contributes significantly to undergraduate students as well as graduate students, in order to understand problems of this type, which are well adopted in university courses. 


\section{References}

[1] J. D. Jackson. Classical Electrodynamics. John Wiley, New York, 1975, 2a. ed.

[2] Goldstein, H., Poole, C., Safko, J., Classical Mechanics, (3rd Edition, New York, 2000).

[3] Sakurai, J. J. (1994). Modern Quantum Mechanics. Addison Wesley.

[4] Arnold, V. I., Mathematical Methods of Classical Mechanics, ( $2^{\circ}$ ed., Springer-Verlag, New York, 1989).

[5] Philip M Morse, Herman Feshbach, (New York: McGraw-Hill, 1953).

[6] E. M. Purcell, Electricity and Magnetism, (2 ${ }^{\mathrm{a}}$ ed., McGraw-Hill, 1985).
[7] Griffiths, D. J., Eletrodinâmica, (3 ${ }^{\mathrm{a}}$ ed Pearson Addison Wesley, 2011).

[8] S. Mohammadi. Electro-Gravitational Effect. International Journal of Science, Technology and Society. Vol. 3, No. 4, 2015.

[9] J. D. Jackson, Am. J. Phys. 67, 107 (1999).

[10] S. D. Deines, Functional Basic Units of Physics and Reference Frames That Preserve Maxwell's Equations. International Journal of Applied Mathematics and Theoretical Physics. Vol. 2, No. 4, 2016.

[11] H. D. Young \& R. A. Freedman, Física III: Eletromagnetismo, (12 . ed., Pearson, São Paulo, Brasil, 2009).

[12] H. Anton, C. Rorres, Elementary Linear Algebra: Applications Version, (John Wiley\&Sons, 2000). 